\title{
ANÁLISE DA QUALIDADE DA ÁGUA POR METODOLOGIA ESTATÍSTICA MULTIVARIADA NA REPRESA VELHA (CEPTA/IBAMA/PIRASSUNUNGA/SP)
}

\section{WATER QUALITY ANALYSIS USING MULTI'-VARIATE STATISTICAL METHODOLOGY IN THE VELHA RESERVOIR (CEPTA/IBAMA/PIRASSUNUNGA/SP)}

\author{
Bufon, A.G.M. ${ }^{1}$; Landim, P.M.B. ${ }^{2}$ \\ ${ }^{1}$ Curso de Pós-Graduação em Geociências e Meio Ambiente, Área de \\ Concentração Geociências e Meio Ambiente, Instituto de Geociências e Ciências \\ Exatas, UNESP. abufon@bol.com.br; \\ ${ }^{2}$ Departamento de Geologia Aplicada, Instituto de Geociências e Ciências Exatas, \\ UNESP.plandim@,rc.unesp.br.
}

\section{RESUMO}

Inúmeras questões de difíceis soluções surgem durante as fases de planejamento, construção e pós-construção de reservatórios que necessitam serem abordadas de forma interdisciplinar, destacando-se dentre estas o processo de deposição de sedimentos. Com a finalidade de analisar o comportamento deposicional de um conjunto de variáveis em represa, este trabalho foi executado, durante os anos de 1998 e 2000, na represa Velha, no Centro de Pesquisa e Gestão de Recursos Pesqueiros Continentais (CEPTA/IBAMA), município de Pirassununga (SP). Esta represa abastece 128 tanques e viveiros para piscicultura, onde foram estabelecidos 6 pontos de coletas de amostras de água, realizadas nos períodos seco e chuvoso. Algumas variáveis quanto à qualidade das águas foram determinadas nas amostras coletadas. Os dados foram submetidos ao método estatístico multivariado da análise de agrupamentos demonstrando que no período seco, os parâmetros tiveram comportamento diferente daquele encontrado para os mesmos parâmetros no período chuvoso. Neste último, apesar do número de agrupamentos ser o mesmo do período seco, ocorrem alterações quanto à natureza dos parâmetros agrupados. Assim, os radicais fosfatos e fósforo total se relacionam diretamente com o material de suspensão no período seco, o mesmo não observado no período chuvoso. $\mathrm{O}$ efeito deposicional neste foi mais disperso entre os pontos de coleta em relação àquele verificado no período seco.

Palavras-chave: Análise multivariada. Aspectos limnológicos. Análise de agrupamentos. Represa. 


\section{ABSTRACT}

Innumerable difficult questions appear during the planning, construction and post-construction phases of reservoirs which must be approached from an interdisciplinary perspective, with the sediment deposition process being particularly important. With the purpose of analyzing the limnological depositional behavior of a set of variables, this study was carried out during the years 1998 and 2000, in the "Velha" reservoir, the Center for Research and Management of Continental Fishing Resources (CEPTA/IBAMA), in the city of Pirassununga (SP). This dam supplies 128 tanks and fisheries for pisciculture, where 6 water sampling points were established; samples were collected in dry as well as the rainy seasons. Some water quality variables were determined in the samples collected. The data was submitted to the statistical multivariate method of cluster analysis demonstrating that in the dry season, the parameters behaved differently when compared to the same parameters in the rainy season. In the latter, although the number of groupings was the same as for the dry period, alterations in the nature of the grouped parameters were observed. Thus, the phosphate radicals and total phosphorus were directly related to the suspended material in the water of the reservoir studied in the dry season, while the same was not observed in the rainy season. The depositional effect in the rainy season was more disperse among the collection points compared to that verified in the dry period.

Key-words: Multivariate analysis. Limnological aspects. Cluster analysis. Dam

\section{INTRODUÇÃO}

Reservatórios são construídos para vários propósitos e, entre eles se destacam aqueles para abastecimento de água, irrigação, geração de energia, prevenção de enchentes e recreação. São formados com o barramento de um vale natural com formação artificial de lagos, associados a uma bacia de drenagem natural, e com as vazões de fluentes sujeitas a controle. Os reservatórios, construídos sobre o curso de um rio, apresentam uma elevação na taxa de sedimentação, motivados pela diminuição da velocidade do fluxo ao ser barrado. Isto ocorre desde a entrada no reservatório, onde é maior a deposição, até o contato com a barragem, principalmente na direção natural do antigo perfil longitudinal do curso de água (PONCE, 1986).

No caso de reservatórios destinados ao abastecimento de água ou à geração de energia elétrica, o controle da vazão permite que o nível de água seja alterado com bastante rapidez, provocando mudanças nas características das margens, com marcante impacto nos habitats aquáticos. Correntes de densidade que ocorrem dentro dos reservatórios podem carrear sedimentos que se depositam em áreas que anteriormente eram varridas pelo escoamento do rio. Os padrões de estratificação 
térmica e química, particularmente na proximidade das barragens, também são marcadamente diferentes das ocorrências em corpos naturais (NOGUEIRA, 1991). Em face desta problemática o manejo de reservatórios torna-se de extrema importância e no intuito de melhor entender os processos deposicionais, um grande número de variáveis acaba sendo coletado. Freqüentemente, porém, não se consegue entender a contento o comportamento complexo desse conjunto de variáveis.

Análise de agrupamentos é um termo usado para descrever diversas técnicas numéricas cujo propósito fundamental é classificar os valores de uma matriz de dados sob estudo em grupos discretos. A técnica classificatória multivariada da análise de agrupamentos pode ser utilizada quando se deseja explorar as similaridades entre indivíduos (modo Q) ou entre variáveis (modo R) definindo-os em grupos, considerando simultaneamente, no primeiro caso todas as variáveis medidas em cada indivíduo e, no segundo, todos os indivíduos nos quais foram feitas as mesmas mensurações (LANDIM, 2002).

Agrupar objetos consiste em reconhecer entre eles um grau de similaridade suficiente para reuni-los num mesmo conjunto. A escolha do método de agrupamento é tão crítica quanto à escolha do coeficiente de associação. Dele dependerá a correta classificação de uma amostra dentro de um ou outro grupo já formado (VALENTIN, 2000).

Entre os diversos métodos à disposição para a análise de agrupamentos, o mais utilizado é o por agrupamentos hierárquicos. A partir da matriz inicial de dados obtém-se uma matriz simétrica de similaridades e inicia-se a detecção de pares de casos com a mais alta similaridade, ou a mais baixa distância. Para essa combinação, segundo níveis hierárquicos de similaridade, escolhe-se entre os diversos procedimentos aglomerativos de tal modo que cada ciclo de agrupamento obedeça a uma ordem sucessiva no sentido do decréscimo de similaridade.

Embora diversas medidas de similaridade tenham sido propostas, somente duas são geralmente usadas: coeficiente de correlação e coeficiente de distância. Se as variáveis forem padronizadas a partir da matriz inicial de dados, dando o mesmo peso a cada uma delas, qualquer um desses coeficientes poderá ser diretamente transformado no outro.

$\mathrm{Na}$ matriz inicial de coeficientes de similaridade estes representam o grau de semelhança entre pares de objetos e os mesmos deverão ser arranjados de acordo com os respectivos graus de similaridade de modo a ficarem agrupados segundo uma disposição hierárquica. Os resultados quando organizados em gráfico, do tipo dendrograma, mostrarão as relações das amostras agrupadas.

Várias técnicas de agrupamentos têm sido propostas, e os métodos mais comumente usados são: "ligação simples" (single linkage method ou nearest neighbor); "ligação completa" (complete linkage method ou farthest neighbor); "agrupamento pareado proporcionalmente ponderado" (weighted pair-group method, WPGM); "agrupamento pareado igualmente ponderado" (unweighted pair- 


\begin{tabular}{|l|r|}
\hline Recebido em: 13/01/2006 & HOLOS Environment, v.7 n.1, 2007 - P. 45 \\
\hline Liberado para Publicação em: 05/03/2007 & ISSN:1519-8421 (CD-ROM) / ISSN:1519-8634 (ON-LINE) \\
\hline
\end{tabular}

group method, UPGM); "variância mínima" (minimum variance clustering ou método de Ward).

Existindo diversas técnicas para a análise de agrupamentos e não havendo testes estatísticos válidos para os resultados obtidos, o pesquisador geralmente fica em dúvida sobre qual método aplicar. Entendendo que esta análise sempre deve ser aplicada com caráter introdutório, e nesse sentido tem o seu mérito, a consideração de ordem pragmática a ser adotada é que o melhor método é aquele que fornece os resultados mais coerentes com a realidade em estudo. Aplicações desta metodologia têm mostrado que os métodos pareados igualmente ponderados são superiores aos demais e que o coeficiente distância usualmente agrupa melhor espécimes ou amostras enquanto o coeficiente de correlação é recomendado para o agrupamento entre variáveis.

A partir destas considerações, procurou-se neste trabalho verificar se haveria diferenças no comportamento de variáveis físicas, químicas e biológicas, encontradas em corpos de água barrados, durante os períodos seco e chuvoso. Para tanto foi utilizada a metodologia estatística multivariada da análise de agrupamentos (cluster analysis), por ser um método que apresenta um resultado gráfico de fácil entendimento e representativo do relacionamento multidimensional entre variáveis. Este procedimento foi realizado com o objetivo de identificar se está ocorrendo estratificação térmica e se a represa é oligotrófica, mesotrófica, eutrófica e hipereutrófica.

\section{MATERIAIS E MÉTODOS}

\section{1. Área de Estudo, Pontos de Coleta e Amostragem}

O local escolhido foi à represa Velha, que é utilizada para abastecimento de uma unidade de pesquisa em piscicultura tropical, ou seja, o Centro de Pesquisa e Gestão de Recursos Pesqueiros Continentais (CEPTA/IBAMA/SP), localizado no Município de Pirassununga, Estado de São Paulo, Brasil. Possui longa tradição no desenvolvimento da piscicultura, tendo sido o Centro Latino Americano de Treinamento em Aqüicultura (1975-1988). Atualmente é uma unidade de pesquisa, com jurisdição em todo o território nacional, tendo a missão de gerar, adaptar e difundir conhecimentos científicos, tecnológicos e ambientais para o ordenamento do uso racional dos ecossistemas aquáticos continentais, com ênfase nos recursos ictícos, visando à execução das políticas ambientais nacionais, nas atribuições federais permanentes, para a melhoria da qualidade de vida (Figura 1). 


\begin{tabular}{|l|r|}
\hline Recebido em: $13 / 01 / 2006$ & HOLOS Environment, v.7 n.1, 2007 - P. 46 \\
\hline Liberado para Publicação em: 05/03/2007 & ISSN:1519-8421 (CD-ROM) / ISSN:1519-8634 (ON-LINE) \\
\hline
\end{tabular}

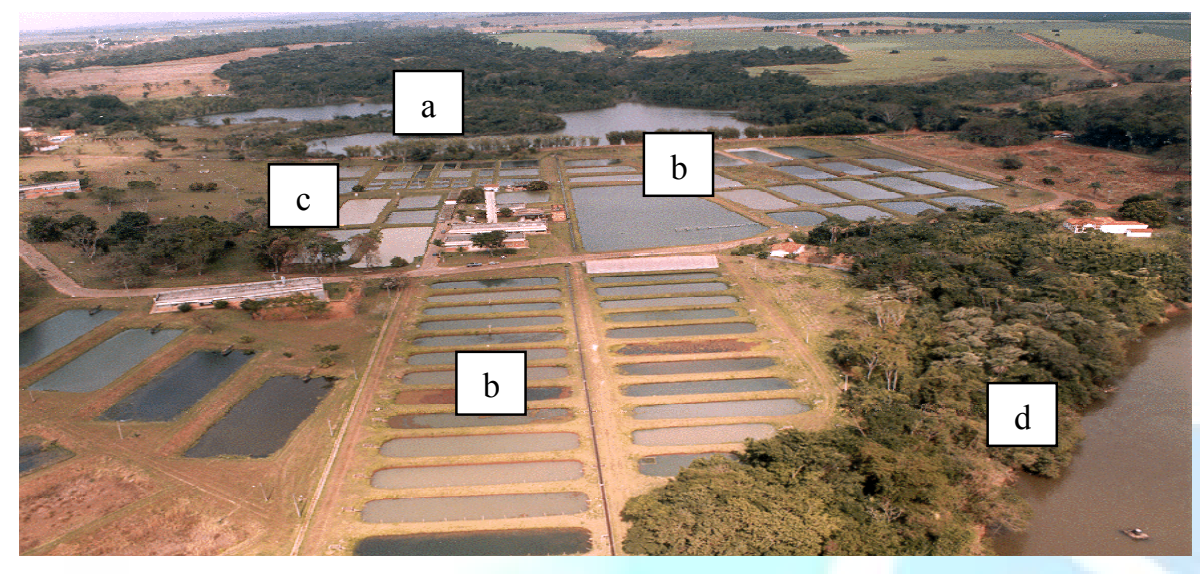

Figura 1. Vista aérea da microbacia do córrego da Barrinha, mostrando a represa Velha.

Legenda: (a) - represa Velha, (b) - tanques e viveiros de criação de peixes, (c) - laboratórios e (d) - rio Mogi-Guaçu. (Fonte: BUFON, 1999).

As amostras foram colhidas, conforme a metodologia descrita por Maricato (1994) e Leite (1998), com periodicidade mensal, nos períodos seco e chuvoso. No período seco as amostras foram coletadas nos meses de junho, julho, agosto e outubro. No período chuvoso nos meses de dezembro, janeiro, fevereiro e abril.

Para as análises das variáveis, as coletas foram feitas em 6 (seis) estações dentro da represa. Em cada ponto de coleta foi instalada verticalmente uma câmara de sedimentação, com altura de $70 \%$ da profundidade total (Figura 2). Algumas amostras como oxigênio dissolvido, temperatura da água e condutividade elétrica foram colhidas na superfície $(0,10 \mathrm{~m})$, no meio (medida relativa à profundidade) e no fundo $(0,10 \mathrm{~m}$ do sedimento). As amostras foram acondicionadas em frascos de vidro de coloração âmbar e mantidas em caixa de isopor contendo blocos de gelo, e levadas ao laboratório, onde as sub-amostras foram separadas em frascos de polietileno, datados, identificados e, se necessário, congeladas para posterior processamento e análises. 


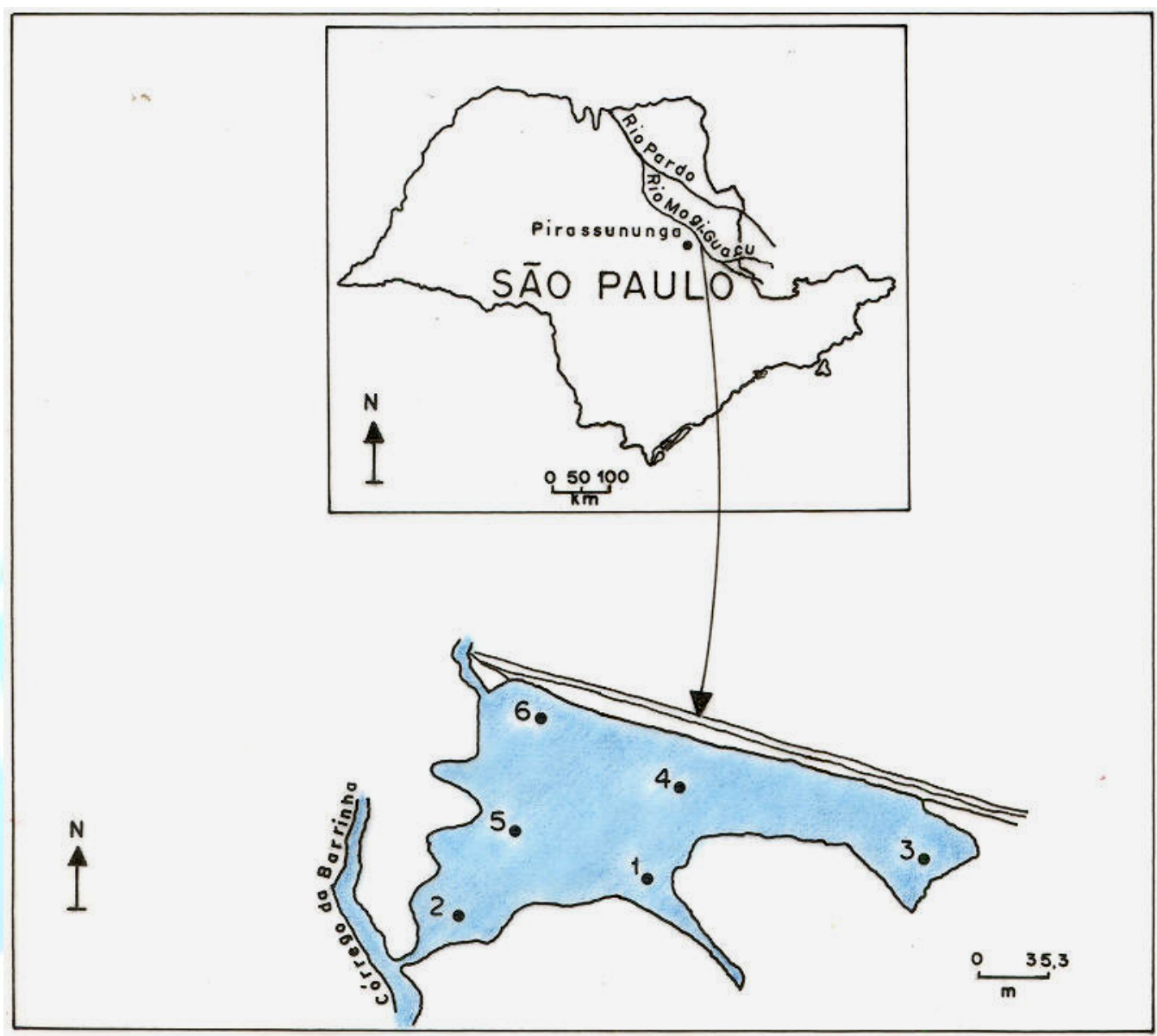

Figura 2. Croqui da represa Velha mostrando os pontos de amostragens. Fonte: Bufon (2002).

Legenda: Pontos - 1 - antigo cercado da represa, 2 - entrada do córrego da Barrinha dentro da represa, 3 - antigo banco de macrófitas, 4 - barragem, 5 meio da represa, 6 - antigo local dos tanques-rede.

\subsection{Metodologia das Análises das Variáveis Físicas, Químicas e Biológica}

A metodologia usada para as análises da água encontra-se, de forma resumida, na Tabela 1. 
Tabela 1 - Metodologias e equipamentos utilizados nas análises das principais variáveis que identificam a qualidade da água da área em estudo.

\begin{tabular}{|c|c|c|c|}
\hline Variáveis & Equipamentos & Métodos & Autores \\
\hline $\begin{array}{l}\text { Temperatura da } \\
\text { água }\end{array}$ & Horiba-U-10 & & \\
\hline $\begin{array}{l}\text { Oxigênio } \\
\text { dissolvido }\end{array}$ & Horiba-U-10 & & \\
\hline Condutividade & Horiba-U-10 & & \\
\hline Transparência & Disco de Secchi & & \\
\hline $\mathrm{pH}$ & Horiba-U-10 & & \\
\hline Alcalinidade & & titulométrico & Boyd (1981) \\
\hline Nitrito e Nitrato & & $\begin{array}{c}\text { espectrofotométrico- } \\
\text { colorimétrico }\end{array}$ & $\begin{array}{l}\text { Mackereth et } \\
\text { al. (1978) }\end{array}$ \\
\hline Amônia & & $\begin{array}{l}\text { espectrofotométrico- } \\
\text { colorimétrico }\end{array}$ & $\begin{array}{l}\text { Koroleff } \\
(1976)\end{array}$ \\
\hline Nitrogênio total & & $\begin{array}{l}\text { espectrofotométrico- } \\
\text { colorimétrico }\end{array}$ & $\begin{array}{l}\text { Mackereth et } \\
\text { al. (1978) }\end{array}$ \\
\hline Fósforo total & & $\begin{array}{l}\text { espectrofotométrico- } \\
\text { colorimétrico }\end{array}$ & $\begin{array}{l}\text { Golterman et } \\
\text { al. (1978) }\end{array}$ \\
\hline $\begin{array}{l}\text { Fosfato total } \\
\text { dissolvido, } \\
\text { fosfato } \\
\text { inorgânico e } \\
\text { silicato reativo } \\
\end{array}$ & & $\begin{array}{l}\text { espectrofotométrico- } \\
\text { colorimétrico }\end{array}$ & $\begin{array}{l}\text { Strickland e } \\
\text { Parsons } \\
(1960) ; \\
\text { Golterman et } \\
\text { al. (1978) }\end{array}$ \\
\hline $\begin{array}{l}\text { Material em } \\
\text { suspensão }\end{array}$ & & gravimétrico & $\begin{array}{l}\text { Wetzel e } \\
\text { Likens (1991) }\end{array}$ \\
\hline Pigmento total & & $\begin{array}{l}\text { espectrofotométrico- } \\
\text { colorimétrico }\end{array}$ & $\begin{array}{l}\text { Marker et al. } \\
(1980)\end{array}$ \\
\hline $\begin{array}{c}\text { Análise } \\
\text { estatística }\end{array}$ & MVSP 3.11 & $\begin{array}{c}\text { Análise de } \\
\text { Agrupamentos }\end{array}$ & Kovach (1999) \\
\hline
\end{tabular}

\subsection{Taxa de Sedimentação de Material Particulado e Nutriente nas Câmaras de Sedimentação e Balanço de Massa}

Para avaliar a deposição de material em suspensão na represa, foram utilizadas, como já mencionado, câmaras de sedimentação (Figura 3) confeccionadas de tubos de PVC de $10 \mathrm{~cm}$ de diâmetro por $30 \mathrm{~cm}$ de comprimento, o que representa uma proporção aproximada de comprimento/diâmetro de 3:1 (MARICATO, 1994). 


\begin{tabular}{|l|r|}
\hline Recebido em: 13/01/2006 & HOLOS Environment, v.7 n.1, 2007 - P. 49 \\
\hline Liberado para Publicação em: 05/03/2007 & ISSN:1519-8421 (CD-ROM) / ISSN:1519-8634 (ON-LINE) \\
\hline
\end{tabular}

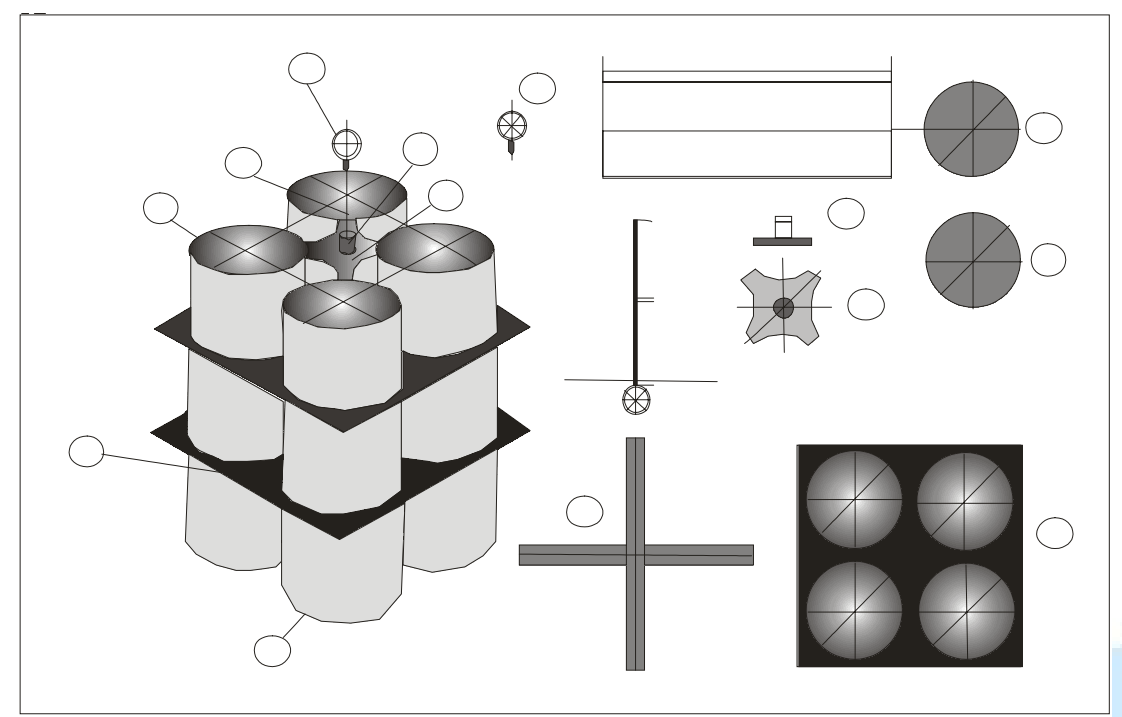

Figura 3. Esquema das câmaras de sedimentação. 1- Abertura da câmara; 2 - fundo da câmara; 3 - suporte das câmaras; 4 - Rosca de trava; 5 Trava das câmaras; 6 - Parafuso da trava das câmaras; 7 - Alça de amarração.

Antes da colocação, as câmaras foram preenchidas com água destilada, evitando-se assim a deposição de material antes do início do experimento. Posteriormente, as mesmas foram colocadas nas profundidades estabelecidas, sendo amarradas a um flutuador de madeira com bóias, o qual foi ancorado em cada ponto de amostragem, permitindo, desta forma, avaliar a diferença espacial na sedimentação do material produzido, bem como as contribuições do principal tributário. O tempo de exposição foi de $24 \mathrm{hs}$, ciclo diário (LEITE, 1998), tempo este suficiente para a acumulação de "tripton" nos coletores. Após o período de incubação, o conteúdo da câmara foi recolhido em galões e levados ao laboratório para a análise da quantidade de material em suspensão (total, inorgânico e orgânico), da concentração de nutrientes totais e dissolvidos, dos pigmentos totais e calculada a taxa de sedimentação.

Os resultados encontrados foram analisados estatisticamente com a análise de agrupamentos (cluster analysis), utilizando o software MVSP 3.11 (KOVACH, 1999). Para essa avaliação foram aplicadas análises de agrupamentos pelo método "agrupamento pareado igualmente ponderado/UPGM", com o coeficiente de correlação de Pearson, como índice de similaridade, pois o interesse era verificar o relacionamento entre variáveis. Preliminarmente essas matrizes de dados tiveram que ser transpostas, não havendo necessidade de padronização para os valores das variáveis. 


\section{RESULTADOS E DISCUSSÃO}

Os resultados dos parâmetros (Tabelas 2 e 3) foram encontrados por Bufon (2002)

Tabela 2 - Dados das análises físicos, químicos e biológico da represa Velha CEPTA/IBAMA no mês de julho de 1998 (período seco).

\begin{tabular}{|c|c|c|c|c|c|c|}
\hline Elementos & $\overline{P 1}$ & $\overline{\mathbf{P 2}}$ & $\overline{\mathbf{P 3}}$ & $\overline{\mathbf{P 4}}$ & $\overline{\mathrm{P5}}$ & $\overline{P 6}$ \\
\hline Alcalinidade $\left(\mathrm{mg} . \mathrm{L}^{-1}\right)$ & 5,00 & 7,00 & 6,00 & 12,00 & 13,00 & 10,00 \\
\hline Amônia $\left(\mu \mathrm{g} . \mathrm{L}^{-1}\right)$ & 32,30 & 95,94 & 68,74 & 71,82 & 68,23 & 46,16 \\
\hline Condutividade elétrica na média prof. $\left(\mu \mathrm{S} . \mathrm{cm}^{-1}\right)$ & 10,00 & 10,00 & 10,00 & 10,00 & 5,00 & 10,00 \\
\hline Condutividade elétrica na superfície $\left(\mu \mathrm{S}_{\mathrm{S}} \mathrm{cm}^{-1}\right)$ & 10,00 & 10,00 & 10,00 & 10,00 & 5,00 & 10,00 \\
\hline Condutividade elétrica no fundo $\left(\mu \mathrm{S} . \mathrm{cm}^{-1}\right)$ & 18,00 & 15,00 & 18,00 & 15,00 & 14,00 & 15,00 \\
\hline Fosfato inorgânico $\left(\mu \mathrm{g} . \mathrm{L}^{-1}\right)$ & 9,13 & 4,65 & 5,59 & 6,22 & 4,96 & 4,65 \\
\hline Fosfato total dissolvido $\left(\mu \mathrm{g} . \mathrm{L}^{-1}\right)$ & 10,21 & 3,35 & 3,35 & 5,84 & 6,84 & 6,84 \\
\hline Fósforo total $\left(\mu \mathrm{g} . \mathrm{L}^{-1}\right)$ & 26,94 & 24,22 & 26,61 & 24,52 & 27,20 & 27,20 \\
\hline Material em Suspensão Inorgânico (mg. $\left.\mathrm{L}^{-1}\right)$ & 119,90 & 8,70 & 50,40 & 47,60 & 36,40 & 33,60 \\
\hline Material em Suspensão Orgânico $\left(\mathrm{mg} . \mathrm{L}^{-1}\right)$ & 39,80 & 10,90 & 35,30 & 14,30 & 6,40 & 11,80 \\
\hline Material em Suspensão Total $\left(\mathrm{mg} \cdot \mathrm{L}^{-1}\right)$ & 159,70 & 19,60 & 85,70 & 61,90 & 42,90 & 45,40 \\
\hline Nitrato $\left(\mu \mathrm{g} . \mathrm{L}^{-1}\right)$ & 62,49 & 58,69 & 59,77 & 60,32 & 58,69 & 57,60 \\
\hline Nitrito $\left(\mu \mathrm{g} . \mathrm{L}^{-1}\right)$ & 2,69 & 2,69 & 3,29 & 3,29 & 3,29 & 3,59 \\
\hline Nitrogênio total $\left(\mu \mathrm{g} . \mathrm{L}^{-1}\right)$ & 521,66 & 402,63 & 552,89 & 662,89 & 302,81 & 772,88 \\
\hline Oxigênio dissolvido na média prof. (mg. $\left.\mathrm{L}^{-1}\right)$ & 7,40 & 8,00 & 7,40 & 7,70 & 8,50 & 8,10 \\
\hline Oxigênio dissolvido na superfície $\left(\mathrm{mg} \cdot \mathrm{L}^{-1}\right)$ & 8,00 & 8,50 & 8,00 & 9,00 & 8,80 & 8,80 \\
\hline Oxigênio dissolvido no fundo (mg. $\left.\mathrm{L}^{-1}\right)$ & 5,50 & 6,70 & 5,50 & 0,80 & 8,50 & 2,20 \\
\hline $\mathrm{PH}$ & 6,67 & 6,83 & 6,56 & 6,73 & 6,83 & 6,66 \\
\hline Pigmento total $\left(\mu \mathrm{g} . \mathrm{L}^{-1}\right)$ & 20,00 & 20,00 & 40,00 & 20,00 & 20,00 & 20,00 \\
\hline Silicato reativo $\left(\mathrm{mg} \cdot \mathrm{L}^{-1}\right)$ & 4,96 & 3,61 & 2,06 & 5,76 & 1,70 & 1,78 \\
\hline Temperatura da água na média prof. $\left({ }^{\circ} \mathrm{C}\right)$ & 20,00 & 20,00 & 19,90 & 19,80 & 19,50 & 20,00 \\
\hline Temperatura da água na superfície $\left({ }^{\circ} \mathrm{C}\right)$ & 20,00 & 20,00 & 19,90 & 20,00 & 20,00 & 20,00 \\
\hline Temperatura da água no fundo $\left({ }^{\circ} \mathrm{C}\right)$ & 20,00 & 19,50 & 19,90 & 18,50 & 18,80 & 19,00 \\
\hline Transparência da água $(\mathrm{m})$ & 0,90 & 0,80 & 0,80 & 0,93 & 0,90 & 0,80 \\
\hline
\end{tabular}


Tabela 3 - Dados das análises físicos, químicos e biológico da represa Velha CEPTA/IBAMA no mês de fevereiro de 2000 (período chuvoso).

\begin{tabular}{|c|c|c|c|c|c|c|}
\hline Elementos & P1 & $\mathbf{P 2}$ & $\mathbf{P 3}$ & P4 & P5 & P6 \\
\hline Alcalinidade $\left(\mathrm{mg} \cdot \mathrm{L}^{-1}\right)$ & 6,00 & 10,00 & 10,00 & 10,00 & 8,00 & 8,00 \\
\hline Amônia $\left(\mu \mathrm{g} . \mathrm{L}^{-1}\right)$ & 75,41 & 14,34 & 5,10 & 21,53 & 51,80 & 45,65 \\
\hline Condutividade elétrica na média prof. $\left(\mu \mathrm{S} . \mathrm{cm}^{-1}\right)$ & 10,00 & 10,00 & 10,00 & 8,50 & 10,00 & 10,00 \\
\hline Condutividade elétrica na superfície $\left(\mu{\left.\mathrm{S} . \mathrm{cm}^{-1}\right)}\right.$ & 10,00 & 10,00 & 10,00 & 8,50 & 10,00 & 10,00 \\
\hline Condutividade elétrica no fundo $\left(\mu \mathrm{S} . \mathrm{cm}^{-1}\right)$ & 30,00 & 20,00 & 10,00 & 20,00 & 10,00 & 10,00 \\
\hline Fosfato inorgânico $\left(\mu \mathrm{g} . \mathrm{L}^{-1}\right)$ & 1,57 & 0,97 & 0,38 & 0,67 & 31,38 & 1,87 \\
\hline Fosfato total dissolvido $\left(\mu \mathrm{g} \cdot \mathrm{L}^{-1}\right)$ & 11,62 & 5,63 & 5,14 & 8,63 & 57,73 & 3,64 \\
\hline Fósforo total $\left(\mu \mathrm{g} . \mathrm{L}^{-1}\right)$ & 5,14 & 37,34 & 55,82 & 37,93 & 27,80 & 26,31 \\
\hline Material em Suspensão Inorgânico (mg. $\left.\mathrm{L}^{-1}\right)$ & 102,20 & 177,20 & 147,80 & 58,10 & 50,40 & 39,20 \\
\hline Material em Suspensão Orgânico (mg. $\mathrm{L}^{-1}$ ) & 49,70 & 48,30 & 65,80 & 31,50 & 28,70 & 24,50 \\
\hline Material em Suspensão Total (mg. L $\left.^{-1}\right)$ & 152,00 & 225,50 & 213,60 & 89,60 & 79,10 & 63,70 \\
\hline Nitrato $\left(\mu \mathrm{g} . \mathrm{L}^{-1}\right)$ & 8,64 & 9,73 & 6,47 & 5,38 & 5,11 & 4,29 \\
\hline Nitrito $\left(\mu \mathrm{g} \cdot \mathrm{L}^{-1}\right)$ & 0,60 & 2,99 & 2,69 & 2,39 & 3,59 & 2,69 \\
\hline Nitrogênio total $\left(\mu \mathrm{g} . \mathrm{L}^{-1}\right)$ & 1527,21 & 865,25 & 1044,00 & 800,09 & 802,22 & 760,56 \\
\hline Oxigênio dissolvido na média prof. (mg. $\mathrm{L}^{-1}$ ) & 6,20 & 8,50 & 8,00 & 8,50 & 8,00 & 8,70 \\
\hline Oxigênio dissolvido na superfície (mg. $\mathrm{L}^{-1}$ ) & 6,60 & 8,00 & 8,60 & 8,50 & 8,50 & 8,70 \\
\hline Oxigênio dissolvido no fundo (mg. $\left.\mathrm{L}^{-1}\right)$ & 3,00 & 2,00 & 3,00 & 2,00 & 6,50 & 6,00 \\
\hline $\mathrm{pH}$ & 5,24 & 6,17 & 6,44 & 6,20 & 6,57 & 6,16 \\
\hline Pigmento total $\left(\mu \mathrm{g} . \mathrm{L}^{-1}\right)$ & 10,00 & 10,00 & 80,00 & 40,00 & 30,00 & 10,00 \\
\hline Silicato reativo $\left(\mathrm{mg} \cdot \mathrm{L}^{-1}\right)$ & 1,63 & 1,73 & 1,63 & 1,72 & 1,59 & 1,59 \\
\hline Temperatura da água na média prof. $\left({ }^{\circ} \mathrm{C}\right)$ & 19,00 & 19,00 & 20,00 & 21,00 & 21,00 & 21,00 \\
\hline Temperatura da água na superfície $\left({ }^{\circ} \mathrm{C}\right)$ & 19,00 & 19,00 & 20,00 & 21,00 & 21,00 & 21,00 \\
\hline Temperatura da água no fundo $\left({ }^{\circ} \mathrm{C}\right)$ & 19,00 & 19,00 & 20,00 & 21,00 & 21,00 & 21,00 \\
\hline Transparência da água (m) & 0,70 & 0,70 & 0,60 & 0,60 & 0,60 & 0,60 \\
\hline
\end{tabular}

Os desvios padrão encontrados dos resultados obtidos demonstraram que houve diferenças dos mesmos entre os períodos seco e chuvoso, Tabela 4. Os resultados da análise de agrupamentos dos parâmetros analisados se encontram nas Figuras 4 e 5 , respectivamente para os períodos seco e chuvoso.

$\mathrm{Na}$ Tabela 5 estão dispostas as variáveis segundo os 3 grupos indicados no dendrograma apresentado na Figura 4. No grupo 1 constam: oxigênio dissolvido no fundo, amônia, temperatura da água na superfície, oxigênio dissolvido na média profundidade, $\mathrm{pH}$, oxigênio dissolvido na superfície e alcalinidade; o grupo 2 é formado por condutividade elétrica na média profundidade, condutividade elétrica na superfície, temperatura da água na média profundidade, nitrogênio total, transparência da água, silicato reativo, condutividade elétrica no fundo, material em suspensão orgânico, temperatura da água no fundo, material em suspensão inorgânico, material em suspensão total, nitrato, fosfato inorgânico e fosfato total dissolvido; e o grupo 3 é formado por nitrito, fósforo total e pigmento total. 


\begin{tabular}{|l|r|}
\hline Recebido em: 13/01/2006 & HOLOS Environment, v.7 n.1, 2007 - P. 52 \\
\hline Liberado para Publicação em: 05/03/2007 & ISSN:1519-8421 (CD-ROM) / ISSN:1519-8634 (ON-LINE) \\
\hline
\end{tabular}

Tabela 4 - Análises realizadas através das médias e dos desvios padrões nos períodos seco e chuvoso durante os meses e anos de julho de 1998 e fevereiro de 2000.

\begin{tabular}{|c|c|c|c|c|}
\hline \multirow[t]{2}{*}{ Variáveis } & \multirow{2}{*}{$\begin{array}{c}\text { jul/98 } \\
\text { Média } \\
\end{array}$} & \multirow[b]{2}{*}{ D.Padrão } & \multirow{2}{*}{$\begin{array}{l}\text { fev/00 } \\
\text { Media }\end{array}$} & \multirow[b]{2}{*}{ D.Padrão } \\
\hline & & & & \\
\hline Alcalinidade (mg. $\left.\mathrm{L}^{-1}\right)$ & 8.833 & 3.312 & 8.667 & 1.633 \\
\hline Amônia $\left(\mu \mathrm{g} . \mathrm{L}^{-1}\right)$ & 63.865 & 22.112 & 35.638 & 26.565 \\
\hline Condutividade elétrica na média prof. $\left(\mu \mathrm{S} . \mathrm{cm}^{-1}\right)$ & 9.167 & 2.041 & 9.750 & 0.612 \\
\hline Condutividade elétrica na superfície $\left(\mu \mathrm{S} . \mathrm{cm}^{-1}\right)$ & 9.167 & 2.041 & 9.750 & 0.612 \\
\hline Condutividade elétrica no fundo $\left(\mu{\mathrm{S} . \mathrm{cm}^{-1}}^{-1}\right)$ & 15.833 & 1.722 & 16.667 & 8.165 \\
\hline Fosfato inorgânico $\left(\mu \mathrm{g} \cdot \mathrm{L}^{-1}\right)$ & 5.867 & 1.711 & 6.140 & 12.377 \\
\hline Fosfato total dissolvido $\left(\mu \mathrm{g} \cdot \mathrm{L}^{-1}\right)$ & 6.072 & 2.576 & 15.398 & 20.933 \\
\hline Fósforo total $\left(\mu \mathrm{g} . \mathrm{L}^{-1}\right)$ & 26.115 & 1.372 & 31.723 & 16.746 \\
\hline Material em Suspensão Inorgânico (mg. $\left.\mathrm{L}^{-1}\right)$ & 49.433 & 37.549 & 95.817 & 56.669 \\
\hline Material em Suspensão Orgânico (mg. $\left.\mathrm{L}^{-1}\right)$ & 19.750 & 14.094 & 41.417 & 15.854 \\
\hline Material em Suspensão Total $\left(\mathrm{mg} . \mathrm{L}^{-1}\right)$ & 69.200 & 49.465 & 137.250 & 70.564 \\
\hline Nitrato $\left(\mu \mathrm{g} . \mathrm{L}^{-1}\right)$ & 59.593 & 1.706 & 6.603 & 2.146 \\
\hline Nitrito $\left(\mu \mathrm{g} . \mathrm{L}^{-1}\right)$ & 3.140 & 0.367 & 2.492 & 1.012 \\
\hline Nitrogênio total $\left(\mu \mathrm{g} \cdot \mathrm{L}^{-1}\right)$ & 535.960 & 170.217 & 966.555 & 292.488 \\
\hline Oxigênio dissolvido na média prof. (mg. $\mathrm{L}^{-1}$ ) & 7.850 & 0.432 & 7.983 & 0.920 \\
\hline Oxigênio dissolvido na superfície $\left(\mathrm{mg} . \mathrm{L}^{-1}\right)$ & 8.517 & 0.431 & 8.150 & 0.797 \\
\hline Oxigênio dissolvido no fundo $\left(\mathrm{mg} . \mathrm{L}^{-1}\right)$ & 4.867 & 2.864 & 3.750 & 1.994 \\
\hline PH & 6.713 & 0.106 & 6.130 & 0.467 \\
\hline Pigmento total $\left(\mu \mathrm{g} . \mathrm{L}^{-1}\right)$ & 23.333 & 8.165 & 30.000 & 27.568 \\
\hline Silicato reativo $\left(\mathrm{mg} \cdot \mathrm{L}^{-1}\right)$ & 3.312 & 1.750 & 1.648 & 0.062 \\
\hline Temperatura da água na média prof. $\left({ }^{\circ} \mathrm{C}\right)$ & 19.867 & 0.197 & 20.167 & 0.983 \\
\hline Temperatura da água na superfície $\left({ }^{\circ} \mathrm{C}\right)$ & 19.983 & 0.041 & 20.167 & 0.983 \\
\hline Temperatura da água no fundo $\left({ }^{\circ} \mathrm{C}\right)$ & 19.283 & 0.611 & 20.167 & 0.983 \\
\hline Transparência da água (m) & 0.855 & 0.061 & 0.633 & 0.052 \\
\hline
\end{tabular}




\begin{tabular}{|l|r|}
\hline Recebido em: $13 / 01 / 2006$ & HOLOS Environment, v.7 n.1, 2007 - P. 53 \\
\hline Liberado para Publicação em: 05/03/2007 & ISSN:1519-8421 (CD-ROM) / ISSN:1519-8634 (ON-LINE) \\
\hline
\end{tabular}

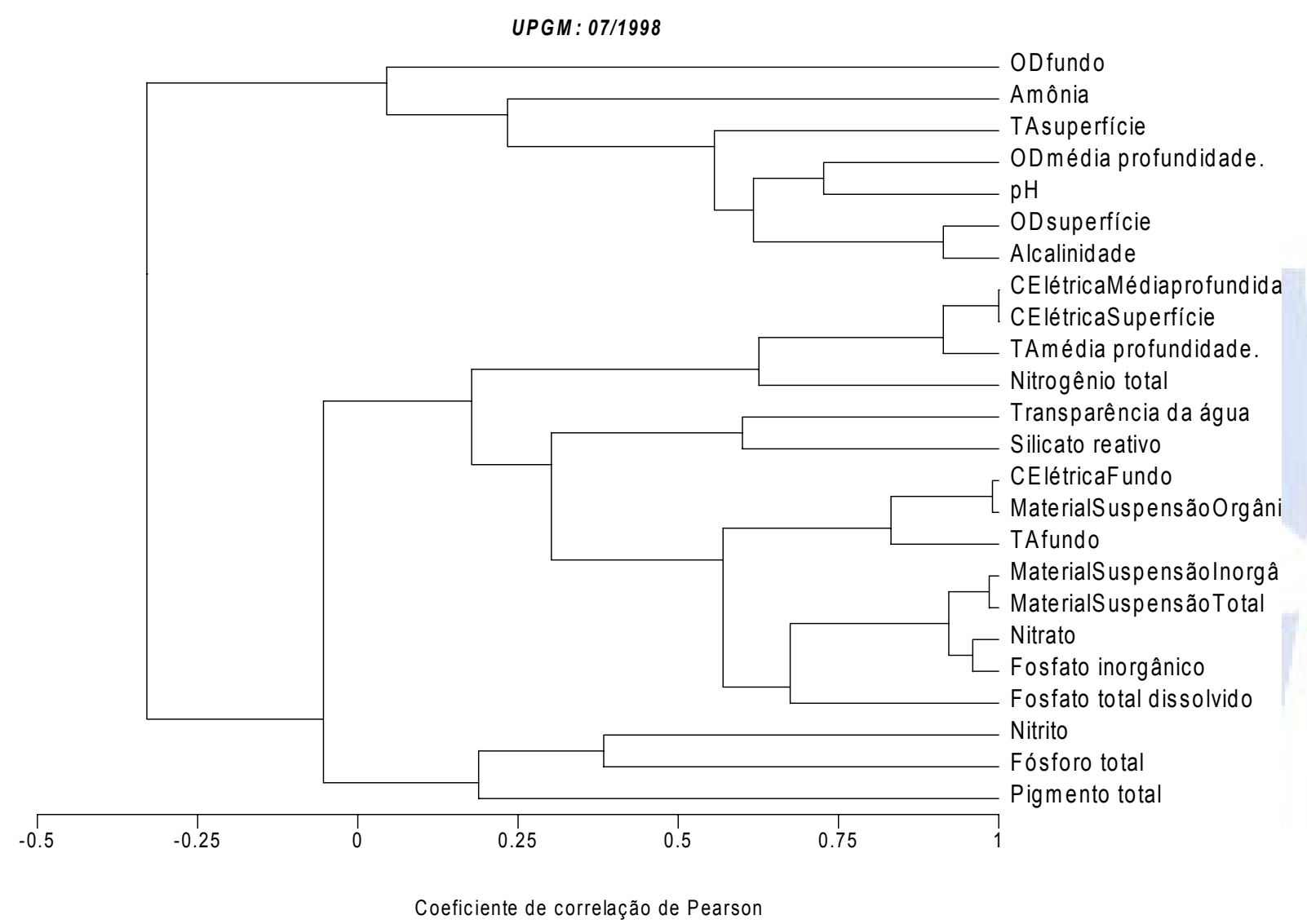

Figura 4. Dendrograma obtido pelo método UPGW, utilizando o coeficiente de correlação de Pearson como medida de similaridade para as variáveis obtidas no período seco (julho/1998). 
Tabela 5 - Grupos formados pela análise de agrupamento dos elementos físicos, químicos e biológico da represa Velha CEPTA/IBAMA no mês de julho de 1998.

\begin{tabular}{|c|c|c|c|c|c|c|}
\hline Elementos & P1 & $\mathbf{P 2}$ & P3 & $\mathbf{P 4}$ & P5 & P6 \\
\hline Oxigênio dissolvido no fundo & 5,50 & 6,70 & 5,50 & 0,80 & 8,50 & 2,20 \\
\hline Amônia & 32,30 & 95,94 & 68,74 & 71,82 & 68,23 & 46,16 \\
\hline Temperatura da água na superfície & 20,00 & 20,00 & 19,90 & 20,00 & 20,00 & 20,00 \\
\hline Oxigênio dissolvido na média profundi. & 7,40 & 8,00 & 7,40 & 7,70 & 8,50 & 8,10 \\
\hline $\mathrm{PH}$ & 6,67 & 6,83 & 6,56 & 6,73 & 6,83 & 6,66 \\
\hline Oxigênio dissolvido na superfície & 8,00 & 8,50 & 8,00 & 9,00 & 8,80 & 8,80 \\
\hline Alcalinidade & 5,00 & 7,00 & 6,00 & 12,00 & 13,00 & 10,00 \\
\hline Condutividade elétrica na média profundi. & 10,00 & 10,00 & 10,00 & 10,00 & 5,00 & 10,00 \\
\hline Condutividade elétrica na superfície & 10,00 & 10,00 & 10,00 & 10,00 & 5,00 & 10,00 \\
\hline Temperatura da água na média profundi. & 20,00 & 20,00 & 19,90 & 19,80 & 19,50 & 20,00 \\
\hline Nitrogênio total & 521,66 & 402,63 & 552,89 & 662,89 & 302,81 & 772,88 \\
\hline Transparência da água & 0,90 & 0,80 & 0,80 & 0,93 & 0,90 & 0,80 \\
\hline Silicato reativo & 4,96 & 3,61 & 2,06 & 5,76 & 1,70 & 1,78 \\
\hline Condutividade elétrica no fundo & 18,00 & 15,00 & 18,00 & 15,00 & 14,00 & 15,00 \\
\hline Material em Suspensão Orgânico & 39,80 & 10,90 & 35,30 & 14,30 & 6,40 & 11,80 \\
\hline Temperatura da água no fundo & 20,00 & 19,50 & 19,90 & 18,50 & 18,80 & 19,00 \\
\hline Material em Suspensão Inorgânico & 119,90 & 8,70 & 50,40 & 47,60 & 36,40 & 33,60 \\
\hline Material em Suspensão Total & 159,70 & 19,60 & 85,70 & 61,90 & 42,90 & 45,40 \\
\hline Nitrato & 62,49 & 58,69 & 59,77 & 60,32 & 58,69 & 57,60 \\
\hline Fosfato inorgânico & 9,13 & 4,65 & 5,59 & 6,22 & 4,96 & 4,65 \\
\hline Fosfato total dissolvido & 10,21 & 3,35 & 3,35 & 5,84 & 6,84 & 6,84 \\
\hline Nitrito & 2,69 & 2,69 & 3,29 & 3,29 & 3,29 & 3,59 \\
\hline Fósforo total & 26,94 & 24,22 & 26,61 & 24,52 & 27,20 & 27,20 \\
\hline Pigmento total & 20,00 & 20,00 & 40,00 & 20,00 & 20,00 & 20,00 \\
\hline
\end{tabular}

O dendrograma exposto na Figura 5 mostra, também 3 agrupamentos e na Tabela 6 está a relação das variáveis. O grupo 1 é constituído por silicato reativo, material em suspensão orgânico, material em suspensão inorgânico, material em suspensão total, condutividade elétrica no fundo, transparência da água, nitrato e nitrogênio total; o grupo 2 por condutividade elétrica na média profundidade, condutividade elétrica na superfície, amônia, fosfato inorgânico, fosfato total dissolvido e oxigênio dissolvido no fundo; e o grupo 3 por temperatura da água no fundo, temperatura da água na média profundidade, temperatura da água na superfície, oxigênio dissolvido na média profundidade, oxigênio dissolvido na superfície, nitrito, $\mathrm{pH}$, fósforo total, alcalinidade e pigmento total. 


\begin{tabular}{|l|r|}
\hline Recebido em: $13 / 01 / 2006$ & HOLOS Environment, v.7 n.1, 2007 - P. 55 \\
\hline Liberado para Publicação em: 05/03/2007 & ISSN:1519-8421 (CD-ROM) / ISSN:1519-8634 (ON-LINE) \\
\hline
\end{tabular}

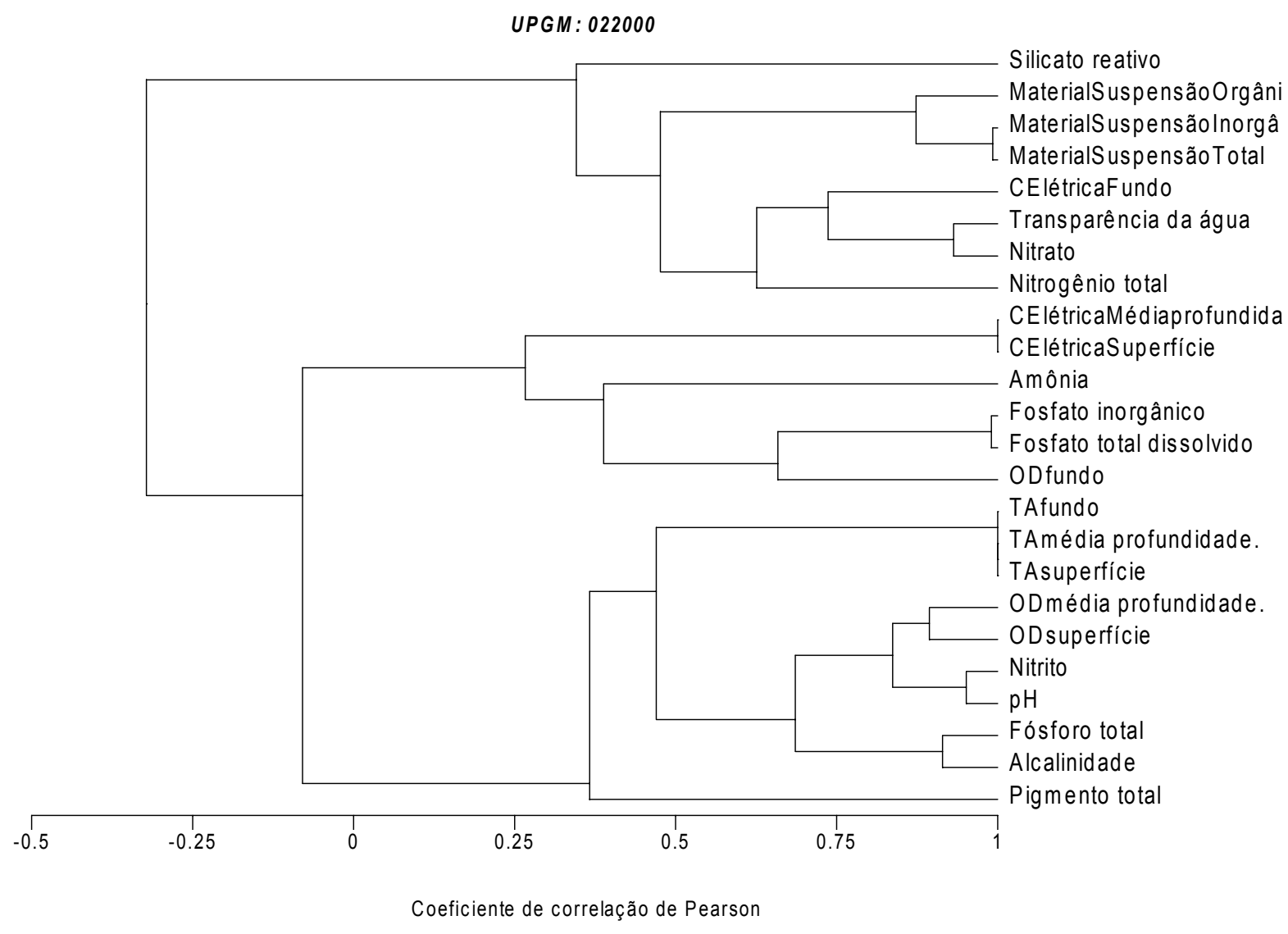

Figura 5. Dendrograma obtido pelo método UPGW, utilizando o coeficiente de correlação de Pearson como medida de similaridade para as variáveis obtidas no período chuvoso (fevereiro de 2000). 
Tabela 6 - Grupos formados pela análise de agrupamento dos elementos físicos, químicos e biológico da represa Velha CEPTA/IBAMA no mês de fevereiro de 2000.

\begin{tabular}{l|r|r|r|r|r|r}
\hline \multicolumn{1}{c|}{ Elementos } & \multicolumn{1}{c|}{ P1 } & \multicolumn{1}{c|}{ P2 } & \multicolumn{1}{c|}{ P3 } & \multicolumn{1}{c|}{ P4 } & \multicolumn{1}{c|}{ P5 } & \multicolumn{1}{c}{ P6 } \\
\hline Silicato reativo & 1,63 & \multicolumn{1}{c}{1,73} & 1,63 & \multicolumn{1}{c}{1,72} & \multicolumn{1}{c}{1,59} & \multicolumn{1}{c}{1,59} \\
\hline Material em Suspensão Orgânico & 49,70 & 48,30 & 65,80 & 31,50 & 28,70 & 24,50 \\
\hline Material em Suspensão Inorgânico & 102,20 & 177,20 & 147,80 & 58,10 & 50,40 & 39,20 \\
\hline Material em Suspensão Total & 152,00 & 225,50 & 213,60 & 89,60 & 79,10 & 63,70 \\
\hline Condutividade elétrica no fundo & 30,00 & 20,00 & 10,00 & 20,00 & 10,00 & 10,00 \\
\hline Transparência da água & 0,70 & 0,70 & 0,60 & 0,60 & 0,60 & 0,60 \\
\hline Nitrato & 8,64 & 9,73 & 6,47 & 5,38 & 5,11 & 4,29 \\
\hline Nitrogênio total & 1527,21 & 865,25 & 1044,00 & 800,09 & 802,22 & 760,56 \\
\hline \hline Condutividade elétrica na média profundi. & 10,00 & 10,00 & 10,00 & 8,50 & 10,00 & 10,00 \\
\hline Condutividade elétrica na superfície & 10,00 & 10,00 & 10,00 & 8,50 & 10,00 & 10,00 \\
\hline Amônia & 75,41 & 14,34 & 5,10 & 21,53 & 51,80 & 45,65 \\
\hline Fosfato inorgânico & 1,57 & 0,97 & 0,38 & 0,67 & 31,38 & 1,87 \\
\hline Fosfato total dissolvido & 11,62 & 5,63 & 5,14 & 8,63 & 57,73 & 3,64 \\
\hline Oxigênio dissolvido no fundo & 3,00 & 2,00 & 3,00 & 2,00 & 6,50 & 6,00 \\
\hline \hline Temperatura da água no fundo & 19,00 & 19,00 & 20,00 & 21,00 & 21,00 & 21,00 \\
\hline Temperatura da água na média profundi. & 19,00 & 19,00 & 20,00 & 21,00 & 21,00 & 21,00 \\
\hline Temperatura da água na superfície & 19,00 & 19,00 & 20,00 & 21,00 & 21,00 & 21,00 \\
\hline Oxigênio dissolvido na média profundi. & 6,20 & 8,50 & 8,00 & 8,50 & 8,00 & 8,70 \\
\hline Oxigênio dissolvido na superfície & 6,60 & 8,00 & 8,60 & 8,50 & 8,50 & 8,70 \\
\hline Nitrito & 0,60 & 2,99 & 2,69 & 2,39 & 3,59 & 2,69 \\
\hline pH & 5,24 & 6,17 & 6,44 & 6,20 & 6,57 & 6,16 \\
\hline Fósforo total & 5,14 & 37,34 & 55,82 & 37,93 & 27,80 & 26,31 \\
\hline Alcalinidade & 6,00 & 10,00 & 10,00 & 10,00 & 8,00 & 8,00 \\
\hline Pigmento total & 10,00 & 10,00 & 80,00 & 40,00 & 30,00 & 10,00 \\
\hline
\end{tabular}

Se confrontadas as tabelas de dados, de números 2 e 3 , com os respectivos dendrogramas, exibidos às Figuras 4 e 5 , pode-se constatar que a análise de agrupamentos mostrou-se uma ferramenta útil para auxiliar no entendimento do processo de sedimentação ocorridos na Represa Velha. Baseando-se nos resultados apresentados por esses dendrogramas algumas conclusões puderam ser feitas.

Com relação ao período seco, ou seja, julho de 1998, com pouca vazão e maior tempo de residência da água na represa, constatou-se três agrupamentos dos elementos físicos, químicos e biológico, mais próximos ao fundo, tendo algumas diferenças nos elementos analisados com relação às médias e desvios padrões como a transparência da água, que possui uma maior visibilidade relacionada com o silicato reativo estando concentrado mais próximo ao fundo, e a temperatura da água na superfície, devido a maior transparência e a um maior aquecimento na superfície, demonstrando a ocorrência da decantação; outros elementos como o nitrato e o nitrito, os quais pelos valores obtidos demonstram pouco consumo, através do fitoplancton, indicando pouca presença; a alcalinidade neste período apresenta-se com maiores valores, os quais representam maiores índices de íons negativos; os valores de oxigênio dissolvido no fundo foram baixos devido a pouca presença de 
fitoplancton; as concentrações da amônia foram altas devido a excreções dos peixes pelas fezes e branqueas e pelos vegetais e animais em decomposição sendo que neste período por ocorrer pouca vazão este material acaba ficando mais concentrado e o pH neste período está no estado tampão como pode ser observado na Tabela 4 , indicando algumas características prováveis de sedimentação.

No período de fevereiro de 2000, chuvoso, com muita vazão e menor tempo de residência da água na represa, observaram-se também três grupos dos elementos próximos ao fundo. Alguns elementos que se destacaram em suspensão nos grupos, eram material em suspensão orgânico, inorgânico e total, que estão correlacionados com a condutividade elétrica no fundo e nitrogênio total, que devido à decomposição dos vegetais e animais carreados pela chuva aumentam os valores. Outros elementos que se destacaram no grupo foram: condutividade elétrica na média profundidade e condutividade elétrica na superfície, sendo estes correlacionados com fosfato inorgânico e fosfato total dissolvido, os quais estão relacionados com vegetais e animais em decomposição, ocorrendo à liberação dos íons e aumentando os valores de condutividade. Para o último grupo observado, os altos valores obtidos de fósforo total estão correlacionados com a temperatura da água na superfície, temperatura da água na média profundidade e temperatura da água no fundo, sendo que através destes elementos ocorre uma proliferação de fitoplancton aumentando as concentrações de pigmentos totais. Devido a esta produção de fitoplancton, os índices de oxigênio dissolvido na média profundidade aumentam, demonstrando que neste período quase não ocorre sedimentação dos elementos em suspensão.

Os resultados aqui encontrados não puderam ser comparados e discutidos com possíveis resultados observados em outra represa quanto à análise de agrupamento dos parâmetros pertinentes à qualidade das águas, pois não foram encontrados em outros trabalhos pertinentes a este assunto.

\section{CONCLUSÕES}

Conclui-se que através da análise de agrupamento que os valores de radicais fosfatos e fósforo total se relacionam diretamente com o material de suspensão nas águas da represa estudada, no período seco e o mesmo não foi observado no período chuvoso. A análise estatística utilizada permitiu concluir que a represa Velha, no município de Pirassununga vem apresentando uma estratificação térmica e ela pode ser considerada oligotrófica.

\section{AGRADECIMENTOS}

Ao Centro de Pesquisa e Gestão de Recursos Pesqueiros Continentais CEPTA/IBAMA, Curso de Pós-Graduação em Geociências e Meio Ambiente da Universidade Estadual Paulista (UNESP), Campus de Rio Claro - SP pelo apoio e 


\begin{tabular}{|l|r|}
\hline Recebido em: $13 / 01 / 2006$ & HOLOS Environment, v.7 n.1, 2007 - P. 58 \\
\hline Liberado para Publicação em: 05/03/2007 & ISSN:1519-8421 (CD-ROM) / ISSN:1519-8634 (ON-LINE) \\
\hline
\end{tabular}

estrutura utilizados neste trabalho e à FAPESP pela bolsa concedida (Processo $\mathrm{n}^{\circ}$ 02/10143-9).

\section{REFERÊNCIAS}

BOYD, C. E. Water quality in warmwater fish culture. [S. 1.]: Auburn University, 1981. 359p.

BUFON, A. G. M. Variação da taxa de sedimentação na represa Velha (CEPTA/IBAMA/SP) e sua influência sobre as características limnológicas do sistema: um estudo de impacto ambiental, Pirassununga/SP. 1999. 80 f. Monografia (Trabalho de Conclusão do Curso (Engenharia de Agrimensura) - Faculdade de Engenharia de Agrimensura de Pirassununga, Pirassununga.1999.

BUFON, A. G. M. Variação temporal e espacial da taxa de sedimentação e das características limnológicas na microbacia do córrego da Barrinha, no município de Pirassununga, SP. 2002. 180 f. Dissertação (Mestrado em Conservação e Manejo de Recursos) - Centro de Estudos Ambientais, Universidade Estadual Paulista, Rio Claro. 2002.

GOLTERMAN, H. L.; CLYMO, R. S.; OHNSTAND, M. A. Methods for physical and chemichal analysis of fresh water. Oxford: Blackwell Scientific Publications, 1978. 213p. (IPB Handbook, n. 8).

KOROLEFF, F. Determination of nutrients. In: GRASSHAFT, K. (Ed.). Methods of seawater omalisys. Verlag: Chemie Weinhein, 1976. p. 117-181.

KOVACH, W. L. MVSP A Multivariate Statistical Package for Windows, ver. 3.11. Kovach Computing Services, Pentraeth, Wales, U.K. 1999.

LANDIM, P. M. B. Análise estatística de dados geológicos multivariados. Rio Claro: Laboratório Geomatemático, UNESP/IGCE/DGA, 2002. 120p. (Texto Didático, 3). Disponível em: http://www.rc.unesp.br/igce/aplicada/textodi.html. Acesso em: 20 de ago. 2003.

LEITE, M. A. Variação espacial e temporal da taxa de sedimentação no Reservatório de Salto Grande (SP) e sua influência sobre as características limnológicas do sistema. 1998. 170 f. Dissertação (Mestrado em Ciências da Engenharia Ambiental)-Escola de Engenharia de São Carlos, Universidade de São Paulo, São Carlos.1998. 


\begin{tabular}{|l|r|}
\hline Recebido em: 13/01/2006 & HOLOS Environment, v.7 n.1, 2007 - P. 59 \\
\hline Liberado para Publicação em: 05/03/2007 & ISSN:1519-8421 (CD-ROM) / ISSN:1519-8634 (ON-LINE) \\
\hline
\end{tabular}

MACKERETH, F. J. H.; HERON, J.; TALLING, F. J. Water analysis some revided methods for limnologists. Ambleside: Freshwater Biological Association, 1978. 120p. (Scientific Publication, n. 36).

MARICATO, F. E. Caracterização físico-química e sedimentação nas regiões de desembocadura dos rios Paranapanema e Taquari, e no reservatório de Jurumirim (Alto Paranapanema-SP). 1994. 205 f. Dissertação (Mestrado em Ciências da Engenharia Ambiental)-Escola de Engenharia de São Carlos, Universidade de São Paulo, São Carlos.1994.

MARKER, A. F. H.; NUSCH, E. A.; RAI; H.; RIEMAN, M. The measurement of photosynthetic pigments in freshwaters and standardization of methods: conchesion and recommendation. Ergebnisse der Limnologie - Archiv Fur Hydrobiologie, Stuttgart, v. 14, p. 91-106, 1980.

NOGUEIRA, V. P. Q. Qualidade da água em lagos e reservatórios. In: PORTO, R. L. L. (Org.). Hidrologia Ambiental: Ed. Universidade São Paulo, Associação Brasileira de Recursos Hídricos, 1991. p. 165-210 (Coleção ABRH de Recursos Hídricos; v. 3).

PONCE, R. J. C. Implantação de uma metodologia de cálculo de assoreamento de reservatórios com aplicação à bacia do rio Sinu - Colômbia. 1986. $172 \mathrm{f}$. Dissertação (Mestrado em Hidráulica e Saneamento) - Escola de Engenharia de São Carlos, Universidade de São Paulo, São Carlos. 1986.

STRICKLAND, J. H. H.; PARSONS, T. R. A manual of sea water analysis, Bulletin. Fisheries Research Board of Canada, Ottawa, v. 125, p. 1-185, 1960.

VALENTIN, J. L. Ecologia numérica: uma introdução à análise multivariada de dados ecológicos. Rio de Janeiro: Interciência, 2000. 117p.

WETZEL, R. G.; LIKENS, G. E. Limnological analysis. New York: Springer Verlag, 1991. 391p. 